\title{
Pythagorean Equation and Special M-Gonal Numbers
}

\author{
M. A. Gopalan ${ }^{1}$, V. Geetha ${ }^{2}$ \\ ${ }^{1}$ (Department of Mathematics, Shrimathi Indira Gandhi College, Trichirappalli-620 002.) \\ ${ }^{2}$ (Department of Mathematics, Cauvery College For Women,Trichirappalli-620 018.)
}

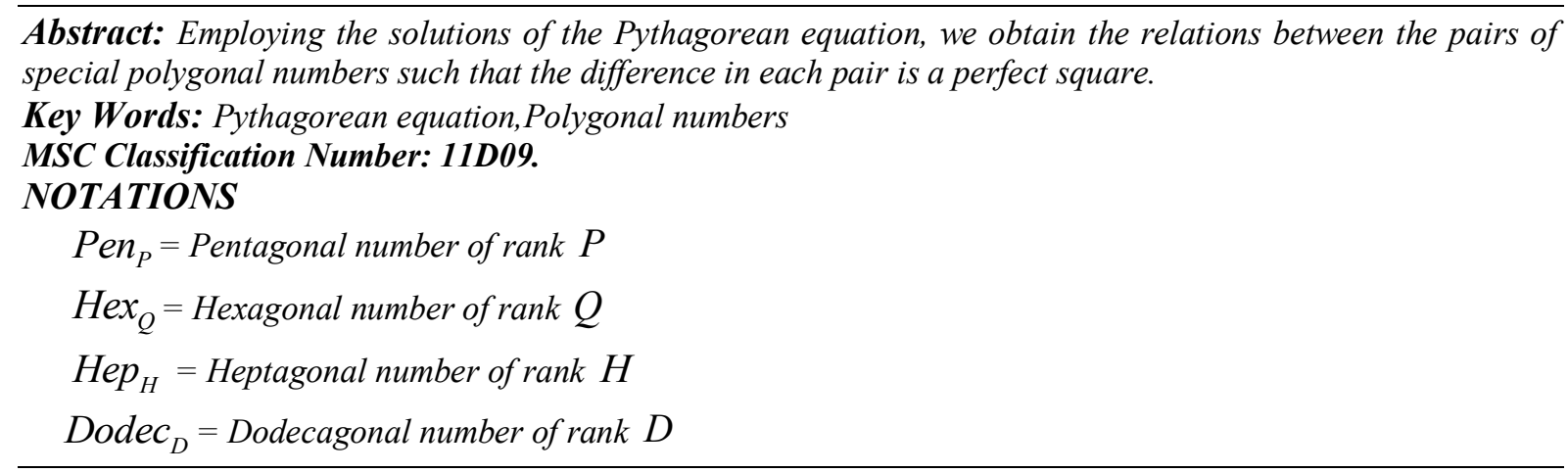

I. Introduction

In $[1,2,4-8,10]$,employing the integral solutions of special binary quadratic Diophantine equation, special patterns of Pythagorean triangles are generated. In [3],the relations among the pairs of special $\mathrm{m}$-gonal numbers generated through the solutions of the binary quadratic equation $y^{2}=2 x^{2}-1$ are determined. In [9], the relations among special figurate numbers through the equation $y^{2}=10 x^{2}+1$ are obtained. In[11],employing the solutions of the Pythagorean equation, and obtain the relations between Triangular number and Pentagonal number, Octagonal number, Hexagonal number, Heptagonal number, Decagonal number, Dodecagonal number , Pentagonal number and Hexagonal number, Octagonal number such that the difference in each pair is a perfect square. In this communication, employing the solutions of the Pythagorean equation, we obtain the relations between the pairs of special polygonal numbers which are not mentioned in [11] such that the difference in each pair is a perfect square.

\section{Method Of Analysis}

Consider the Pythagorean equation

$$
x^{2}+y^{2}=z^{2}
$$

whose solutions are

$$
x=2 r s, y=r^{2}-s^{2}, z=r^{2}+s^{2}
$$

where $r, s$ are non - zero distinct integers and $r>s$.

Case.2.1:

The choice

$$
10 H-3=r^{2}+s^{2}, 18 P-3=r^{2}-s^{2}
$$

in (1) leads to the relation

$$
40 \mathrm{Hep}_{H}-216 \mathrm{Pen}_{P}=\alpha^{2}
$$

From (3), the values of ranks of the Heptagoanl numbers and Pentagonal numbers are respectively given by

$$
P=\frac{r^{2}-s^{2}+3}{18} ; H=\frac{r^{2}+s^{2}+3}{10}
$$

It is seen that $P, H$ are integers for the following choices of $r$ and $s$ namely,
(i) $s=1 ; r=14$
(ii) $s=4 ; r=11$
(iii) $s=15 n-14 ; r=15 n-11$ 
(iv) $s=15 n-4$; $r=15 n+11$

(v) $s=15 n-4 ; r=15 n+29$

For each of the values of $r$ and $s$ the corresponding Pentagonal and Heptagonal numbers satisfying (4) are presented in the Table (1)below.

Table (1)

\begin{tabular}{|c|c|c|c|c|c|}
\hline $\begin{array}{c}\mathrm{S} \\
\mathrm{N} \\
\mathrm{o}\end{array}$ & $P$ & $H$ & $\operatorname{Pen}_{P}$ & $\operatorname{Hep}_{H}$ & $\alpha$ \\
\hline 1 & 6 & 14 & 51 & 469 & 970 \\
\hline 2 & 11 & 20 & 176 & $\frac{1}{2}\left(45 n^{2}-75 n+32\right)\left(225 n^{2}-375 n+15\right.$ & $\left(450 n^{2}-750 n+308\right.$ \\
\hline 3 & $5 n-4$ & $45 n^{2}-75 n+32$ & $\frac{1}{2}\left(75 n^{2}-125 n+52\right)$ & & \\
\hline 4 & $25 n-6$ & $45 n^{2}+21 n+14$ & $\frac{1}{2}\left(1875 n^{2}+875 n+102\right)$ & $\frac{1}{2}\left(45 n^{2}+21 n+14\right)\left(225 n^{2}+105 n\right.$ & $\left(450 n^{2}+210 n+£\right.$ \\
\hline 5 & $55 n+4$ & $45 n^{2}+75 n+86$ & $\frac{1}{2}\left(9075 n^{2}+15125 n+630\right.$ & $\frac{1}{2}\left(45 n^{2}+75 n+86\right)\left(225 n^{2}+375 n+\right.$ & $\left(450 n^{2}+750 n-232\right.$ \\
\hline
\end{tabular}

Case 2.2:

The Choice

$$
5 \mathrm{D}-2=r^{2}-s^{2} ; 12 P-2=r^{2}+s^{2}
$$

in (1) leads to the relation

$$
96 \text { Pen }_{P}-5 \text { Dodec }_{D}=\alpha^{2}
$$

From (5), the values of ranks of the Pentagonal and Dodecagonal numbers are respectively given by,

$$
P=\frac{r^{2}+s^{2}+2}{12}, D=\frac{r^{2}-s^{2}+2}{5}
$$

It is seen that $P$ and $D$ are integers for the following choices of $r$ and $s$ namely,
(i) $s=1 ; r=30 n-27$
(ii) $s=9 ; r=30 n \pm 7$
(iii) $s=9 ; r=30 n \pm 17$
(iv) $s=1 ; r=30 n \pm 3$
(v) $s=11 ; r=30 n \pm 3$
(vi) $s=19 ; r=30 n \pm 3$
(vii) $s=30 n-19 ; r=60 n-27$
(viii) $s=30 n+1 ; r=60 n+3$

For each of the values of $r$ and $s$ the corresponding values of $P$ and $D$ are presented in the Table (2) below.

Table (2)

\begin{tabular}{|c|c|c|}
\hline S.No & $P$ & $D$ \\
\hline 1 & $75 n^{2}-135 n+61$ & $180 n^{2}-324 n+146$ \\
\hline 2 & $75 n^{2} \pm 35 n+11$ & $180 n^{2} \pm 84 n-6$ \\
\hline 3 & $75 n^{2} \pm 85 n+31$ & $180 n^{2} \pm 204 n+42$ \\
\hline 4 & $75 n^{2} \pm 15 n+1$ & $180 n^{2} \pm 36 n+2$ \\
\hline 5 & $75 n^{2} \pm 15 n+11$ & $180 n^{2} \pm 36 n+22$ \\
\hline 6 & $75 n^{2} \pm 15 n+31$ & $180 n^{2} \pm 36 n+70$ \\
\hline
\end{tabular}




\begin{tabular}{|c|c|c|}
\hline 7 & $375 n^{2}-365 n+91$ & $540 n^{2}-420 n+74$ \\
\hline 8 & $375 n^{2}+35 n+1$ & $540 n^{2}+60 n+2$ \\
\hline
\end{tabular}

In Table(3) below represent the corresponding Pentagonal and Dodecagonal numbers are exhibited.

Table(3)

\begin{tabular}{|c|c|c|c|}
\hline $\begin{array}{c}\text { S. } \\
\text { No }\end{array}$ & Pen $_{P}$ & Dodec $_{D}$ & $\alpha$ \\
\hline 1 & $\frac{1}{2}\left(75 n^{2}-135 n+61\right)\left(225 n^{2}-405 n+182\right)$ & $\left(180 n^{2}-324 n+146\right)\left(900 n^{2}-1620 n+726\right)$ & $(60 n-54)$ \\
\hline 2 & $\frac{1}{2}\left(75 n^{2} \pm 35 n+11\right)\left(225 n^{2} \pm 105 n+32\right)$ & $\left(180 n^{2} \pm 84 n-6\right)\left(900 n^{2} \pm 420 n-34\right)$ & $(540 n \pm 126)$ \\
\hline 3 & $\frac{1}{2}\left(75 n^{2} \pm 85 n+31\right)\left(225 n^{2} \pm 255 n+92\right)$ & $\left(180 n^{2} \pm 204 n+42\right)\left(900 n^{2} \pm 1020 n+206\right)$ & $(540 n \pm 306)$ \\
\hline 4 & $\frac{1}{2}\left(75 n^{2} \pm 15 n+1\right)\left(225 n^{2} \pm 45 n+2\right)$ & $\left(180 n^{2} \pm 36 n+2\right)\left(900 n^{2} \pm 180 n+6\right)$ & $(60 n \pm 6)$ \\
\hline 5 & $\frac{1}{2}\left(75 n^{2} \pm 15 n+11\right)\left(225 n^{2} \pm 45 n+32\right)$ & $\left(180 n^{2} \pm 36 n+22\right)\left(900 n^{2} \pm 180 n-114\right)$ & $(660 n \pm 66)$ \\
\hline 6 & $\frac{1}{2}\left(75 n^{2} \pm 15 n+31\right)\left(225 n^{2} \pm 45 n+92\right)$ & $\left(180 n^{2} \pm 36 n+70\right)\left(900 n^{2} \pm 180 n-354\right)$ & $(1140 n \pm 114)$ \\
\hline 7 & $\frac{1}{2}\left(375 n^{2}-365 n+91\right)\left(1125 n^{2}-1095 n+272\right)$ & $\left(540 n^{2}-420 n+74\right)\left(2700 n^{2}-2100 n+366\right)$ & $\left(3600 n^{2}-3900 n+1026\right)$ \\
\hline 8 & $\frac{1}{2}\left(375 n^{2}+35 n+1\right)\left(1125 n^{2}+105 n+2\right)$ & $\left(540 n^{2}+60 n+2\right)\left(2700 n^{2}+300 n+6\right)$ & $\left(3600 n^{2}+300 n+6\right)$ \\
\hline
\end{tabular}

\section{Case2.3:}

The Choice

$$
12 Q-3=r^{2}+s^{2} ; 10 H-3=r^{2}-s^{2}
$$

in (1) leads to the relation

$$
72 \mathrm{Hex}_{Q}-40 \mathrm{Hep}_{H}=\alpha^{2} \text {. }
$$

From (6), the values of ranks of the Hexagonal and Heptagonal numbers are respectively given by

$$
Q=\frac{r^{2}+s^{2}+3}{12} ; H=\frac{r^{2}-s^{2}+3}{10}
$$

which are integers for the following three choices of $r$ and $s$ namely,

$$
\begin{aligned}
& \text { (i) } s=15 n-3 ; r=15 n+6 \\
& \text { (ii) } s=15 m-12 ; r=30 n+15 m-21 \\
& \text { (iii) } s=15 m-12 ; r=30 n+15 m-39
\end{aligned}
$$

For each of the values of $r$ and $s$ the values of $Q$ and $H$ are presented in the Table(4) below

Table(4)

\begin{tabular}{|c|c|c|}
\hline S.No & $Q$ & $H$ \\
\hline 1 & $\frac{1}{12}\left(450 n^{2}+90 n+48\right)$ & $27 n+3$ \\
\hline 2 & $\frac{1}{12}\left(900 n^{2}+900 n m+450 m^{2}-1260 n-990 m+588\right)$ & $90 n^{2}+90 n m-126 n-27 m+30$ \\
\hline 3 & $\frac{1}{12}\left(900 n^{2}+900 n m+450 m^{2}-2340 n-1170 m+1668\right)$ & $90 n^{2}+90 n m-234 n-81 m+138$ \\
\hline
\end{tabular}


In Table(5) below represent the corresponding Hexagonal and Heptagonal numbers are exhibited.

Table(5)

\begin{tabular}{|l|l|l|l|}
\hline S.no & \multicolumn{1}{|c|}{$\operatorname{Hex}_{Q}$} & \multicolumn{1}{c|}{$\mathrm{Hep}_{H}$} & \multicolumn{1}{c|}{$\alpha$} \\
\hline 1 & $\frac{1}{72}\left(450 n^{2}+90 n+48\right)\left(450 n^{2}+90 n+42\right)$ & $(27 n+3)(135 n+12)$ & $\left(450 n^{2}+90 n-36\right)$ \\
\hline 2 & $\frac{1}{72}\left(900 n^{2}+900 m+450 m^{2}-1260 n-990 m+588\right)\left(900 n^{2}+900 m+450 m^{2}-1260 n-990 m+582\right)$ & $\begin{array}{l}\left(90 n^{2}+90 m m-126 n-27 m+30\right) \\
\left(450 n^{2}+450 m-630 n-135 m+147\right)\end{array}$ & $\begin{array}{l}450 m^{2}-990 m-720 n \\
+900 m m+504\end{array}$ \\
\hline 3 & $\frac{1}{72}\left(900 n^{2}+900 n m+450 m^{2}-2340 n-1170 m+1688\right)\left(900 n^{2}+900 n m+450 m^{2}-2340 n-1170 m+1682\right)$ & $\begin{array}{l}\left(90 n^{2}+90 n m-234 n-81 m+138\right) \\
*\end{array}$ & $\begin{array}{l}450 m^{2}-1170 m-720 n \\
+900 n m+936 \\
\left(450 n^{2}+450 n m-1170 n-405 m+687\right)\end{array}$ \\
\hline
\end{tabular}

\section{Conclusion}

One may search for relations among other m-gonal numbers such that the difference in each pair is a perfect square.

\section{References}

[1] M.A.Gopalan and G.Janaki,Observations on $y^{2}=3 x^{2}+1$ Acta Ciencia Indica XXXIVM (2) (2008) $103-106$.

[2] M.A.Gopalan and B.Sivakami,Observations on the integral solutions of $y^{2}=7 x^{2}+1$, Antarctica J. Math. 7(3),(2010) ,291296

[3] M.A.Gopalan and G.Srividhya,Relations among m-gonal numbers through equation $y^{2}=2 x^{2}-1$,Antarctica J. Math. $7(3),(2010), 363-369$

[4] M.A.Gopalan and R.Vijayalakshmi,Special Pythagorean triangles generated through the integral solutions of the equation $y^{2}=\left(k^{2}+1\right) x^{2}+1$,Antarctica J. Math. 7(5),(2010), 503-507

[5] M.A.Gopalan and R.Vijayalakshmi, Observations on the integral solutions of $y^{2}=5 x^{2}+1$, Impact J.Sci.Tech. 4(4),(2010), $125-129$

[6] M.A.Gopalan and R.S.Yamuna, Remarkable observations on the binary quadratic equation $y^{2}=\left(k^{2}+2\right) x^{2}+1$, Impact J.Sci.Tech. 4(4),(2010), 61-65

[7] M.A.Gopalan and G.Sangeetha,A Remarkable observation on the binary quadratic equation $y^{2}=10 x^{2}+1$, Impact J.Sci.Tech. 4 (2010), 103-106

[8] M.A.Gopalan and R.Palanikumar, Observations on $y^{2}=12 x^{2}+1$, Antarctica J. Math. 8(2),(2011),149-152

[9] Manju Somanath,G.Sangeetha and M.A.Gopalan, Relations among special figurate numbers through equation $y^{2}=10 x^{2}+1$, Impact J.Sci.Tech. 5(1),(2011), 57-60

[10] M.A.Gopalan and K.Geetha, Observations on the Hyperbola $y^{2}=18 x^{2}+1$,RETELL, 13(1), Nov.2012,81-83.

[11]. M.A.Gopalan, V.Sangeetha and Manju Somanath, Pythagorean equation and Special m-gonal numbers, sent to Antartica journal 